\title{
Research and application of high concurrent real-time streaming forwarding service under multi-source heterogeneous network
}

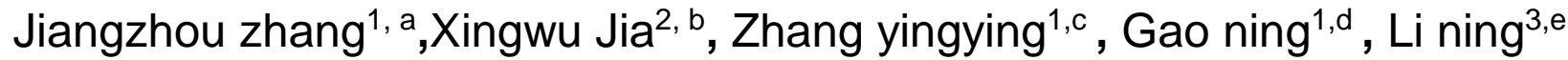 \\ ${ }^{1}$ Information Research Institute, Qilu University of Technology(Shandong Academy of \\ Sciences),Ji'nan City, Shandong, China \\ 2Ji'nan City, Shandong, China \\ ${ }^{3}$ Qilu Institute of Technology, Ji'nan City, Shandong, China \\ azhangjzh@sdas.org, b93671986@qq.com, 'zhangyy@sdas.org, 'dgaon@sdas.org, \\ e1538856724@qq.com
}

Keywords: the real time stream, heterogeneous network

Abstract:In order to solve the real time stream forwarding problem of multi source video on heterogeneous networks, a set of efficient signaling request flow and forwarding algorithm is designed to realize the undifferentiated forwarding service and unified management platform for different vendors and different devices. The protocol stack, scheduling service, resource allocation, multi to many forwarding, variable cache modules are implemented on the platform, and the multi-source heterogeneous video information cascade scheduling is realized.

\section{Introduction}

Video surveillance, as a continuous development and more and more attention, has been widely used in people's life, especially in the field of security has an irreplaceable position. The application of video surveillance system is of positive significance for reducing manpower consumption, saving security funds and weakening the motive of crime.

At present, there are some difficulties in the centralized control and unified use of monitoring equipment because of the various kinds of cameras, different standards, network changes and other factors in the security field. Moreover, the video surveillance system tends to be applied in a large standard and wide range, and the incompatibility between devices becomes more and more obvious. For example, the monitoring systems built with different vendors' terminals are not compatible with each other, and even the products of the same series and different models of some vendors have compatibility problems.

Not only in different industries, but also in the same industry, the video monitoring system is based on a small range of regional deployment, and presents a certain difference. Although the structure forms are basically the same, the specific modules of the system have a deep heterogeneity. It is mainly embodied in 3 aspects: the difference of server function interface in the equipment management layer, the difference of system service type and interface in the system service layer, the difference of flow format and mode.

\section{Research status}

With the continuous development of the Internet and the continuous improvement of network bandwidth, the real-time transmission of streaming media data has become a hot topic in data transmission in recent years.

Due to the lack of authoritative standards that can be followed in the field of security, there are many kinds of video surveillance devices and brands. The video compression transmission standards and transmission protocols adopted by different manufacturers are not uniform and are not compatible with each other. At present, some departments in most cities have established their own independent video surveillance network. Because of the use of different developers, many information isolated islands have been formed. 
The video surveillance system is usually composed of the front camera part, the network transmission part, the back end display part and the control part. The distribution of video streams can be divided into real-time video streaming and non real-time video streaming. The real-time video stream mainly transfers the collected data to the display part in time, which is convenient for users to understand the scene in time and other video image analysis applications. Rather than real-time video streaming is mainly used for video data extraction, on-site forensics and so on.

\section{System architecture}

The system mainly consists of three parts: streaming media forwarding server, central management and scheduling server and client. The streaming media service is responsible for video forwarding, dynamic flow control, coding, and so on. Central management services include security, management scheduling, SIP/RTP protocol cluster and data storage four logic services. The client is mainly viewed as the client. Frequency request's initiation, decoding, application and so on, adopt two modes of C/S and $\mathrm{B} / \mathrm{S}$.

The system mainly studies and analyzes the main business requirements of video monitoring and dynamic scheduling, refining and streamlining, and dividing the requirements logically to achieve modular classification. Then collect, study and analyze all kinds of standards, divide signaling and data flow, and use unified standards to package. On this basis, we collect and study the terminal acquisition equipment of different manufacturers, different modes and different flow formats, and carry out a unified interface package to achieve transparency principle for the subsequent call in the algorithm. Finally, aiming at system design, different functional modules are divided to encapsulate functions.

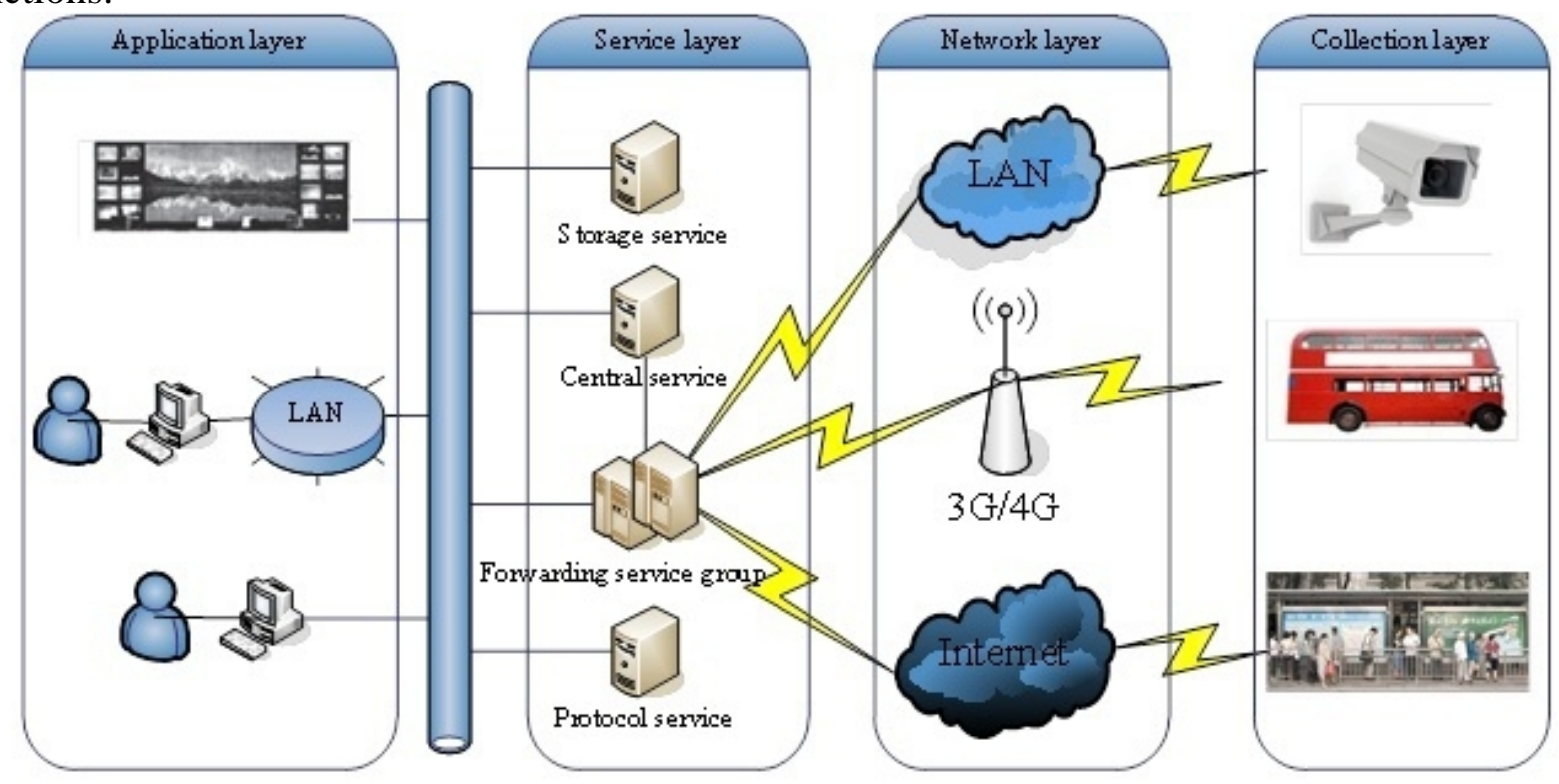

Fig 1

\section{Key technology}

Signaling protocol.Under heterogeneous network environment, it achieves multi-source and non differential transmission. Support 3G/4G network, support routing penetration technology, support internal and external network transformation. It supports multi - mode, multi - vendor devices with no difference transport stream.

The "many to one" forwarding mode saves the network bandwidth of front-end devices. "One" is front-end device to forwarding service, and "multi" means forwarding service to the client. The same device only needs to login once, requesting a video. When multiple users request the same video source, the forward mode is transmitted by one to many modes, which saves the front end bandwidth and achieves no difference transmission. 
Support multilevel forward service. Multiple forwarding services can provide services in parallel, and are managed and dispatched uniformly by the control scheduling service. Both the forwarding service and the client adopt the quantitative caching mechanism to ensure the fluency of the video playback without affecting the timeliness requirements.

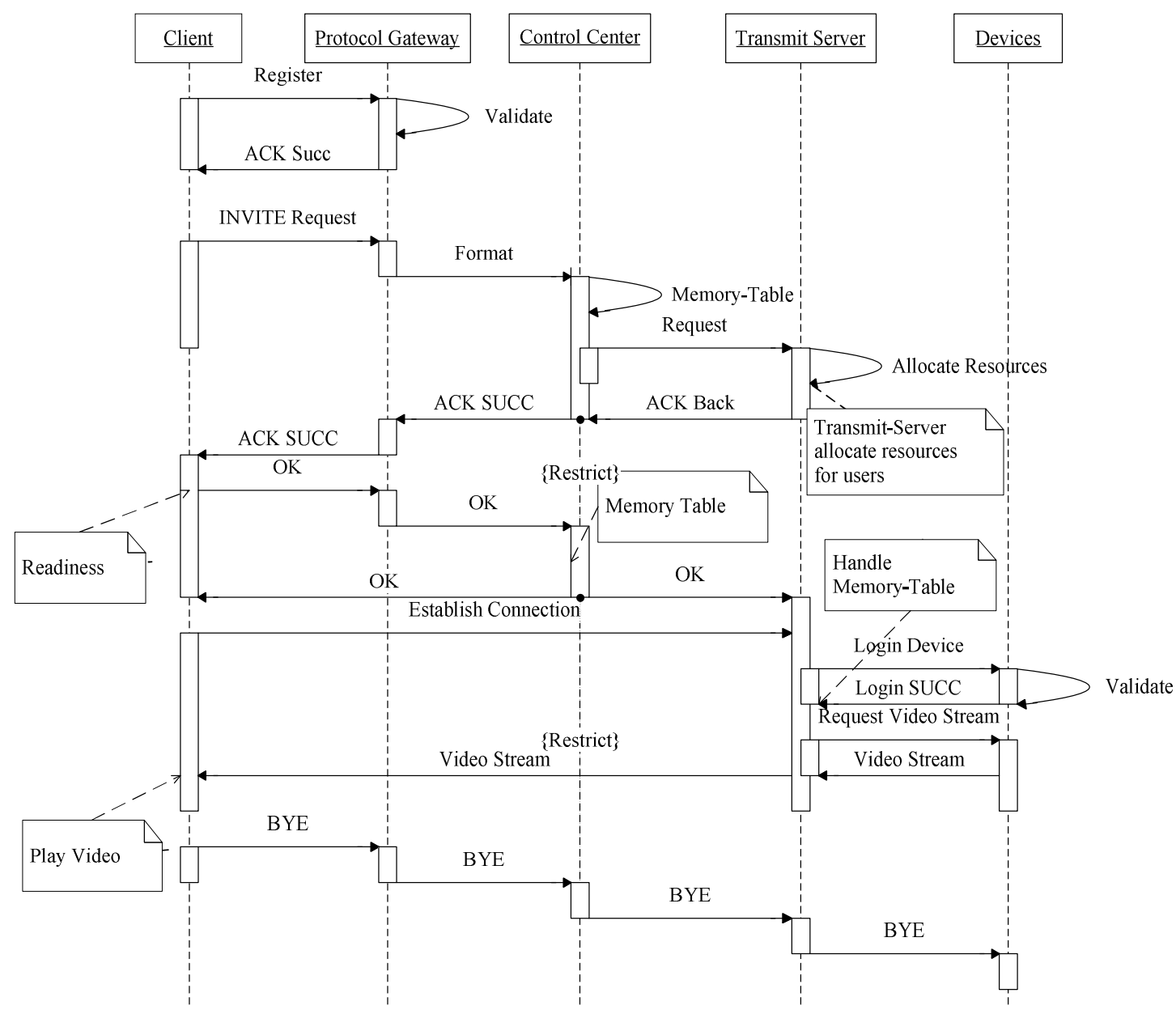

Fig 2

Adaptive multi-source video acquisition and forwarding technology. Research and analyze the main business requirements of video surveillance and dynamic scheduling, from which training and streamlining, and logical division of needs, to achieve modular classification. Collect, study and analyze all kinds of standards, divide signaling and data flow, and use unified standards to package. Collect and study the terminal acquisition equipment of different manufacturers, different modes and different flow formats, and carry out a unified interface package to achieve transparency principle for subsequent calls in the algorithm. Aiming at system design, different functional modules are divided to encapsulate functions.

\begin{tabular}{|c|}
\hline Forwarding scheduling layer \\
\hline Equipment aggregation layer \\
\hline Equipment packaging layer \\
\hline
\end{tabular}

Fig 3
Third layer

Second layer

First layer

The system multi-source video capture is mainly implemented in the streaming media service, and has three layers of encapsulation. The first tier device encapsulation layer, each type of device corresponds to a class, to achieve the device's video read, codec and other functions, and provide services upwards. The second tier device convergence layer, the device packaging layer in the device class to achieve unified and standardized.Third tier forwarding scheduling layer, to achieve user scheduling requests, and through the convergence layer to achieve device access and forwarding. 
Research on dynamic flow control technology. When the compression ratio of different video streams is not consistent and the image quality is different, it is a common problem in the system that how to keep the consistency in high efficiency forwarding service and high efficiency client mode.Because of the different format and quality of terminal sampling devices of different manufacturers and different manufacturers, adaptive dynamic traffic control technology should be adopted. Forwarding service is forwarded according to prior knowledge when forwarding. The link between client and forwarding service is established through pre established links, and the traffic is coordinated through private signaling to achieve dynamic traffic control.

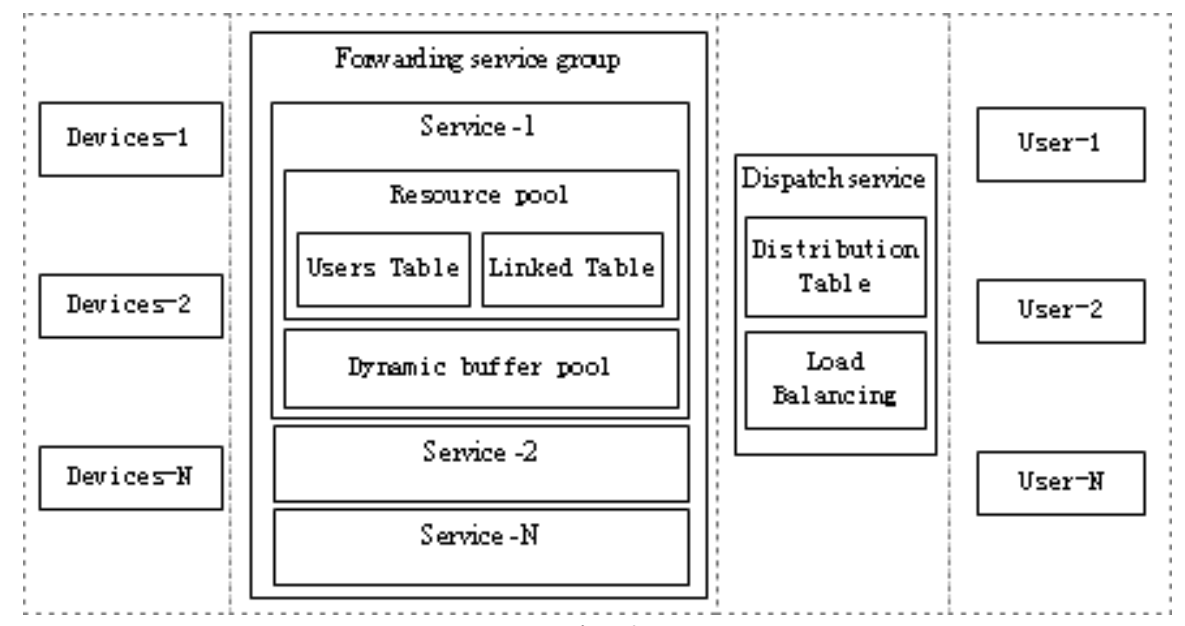

Fig 4

\section{Analysis and summary of the results}

The signaling request flow and forwarding algorithm designed in this paper can solve the problem of real-time stream forwarding in multi source video on heterogeneous networks, and can realize the undifferentiated forwarding service and unified management platform for different vendors and different devices.

\section{References}

[1] Wang Wei Design and implementation of video data stream forwarding in security monitoring system. 2013, Beijing Jiaotong University.

[2] Lv Shaojun and Zhou Yuanping, Real-time streaming media transmission system based on Live555, computer system application, 2015, 24 (1): P, 56-59.

[3] Liu Nan, A flow control method in real-time video transmission,.Journal of Zhengzhou Econom ICS\&Management institute, June 2006,21(2),P84-86.

[4] Cheng deqiang,Flow medium transmission control system base on data package transmitted,Coal science and technology, April 2007,35(4),P52-54.

[5] Ma Zhilong, Research on Quality of ExPerience For Streaming Media Service System,Huazhong university of science\&technology,2009.

[7] Wang Zhao, Research on load balancing strategy of streaming media server cluster, Xi'an University of Posts\&Telecommunications,2017. 